\title{
Propiedades Psicométricas del Trait Meta-Mood Scale (TMMS-24) en Adolescentes de Chiapas, México
}

\author{
Jesús Ocaña Zúñiga \\ Germán Alejandro García Lara \\ Oscar Cruz Pérez
}

Universidad de Ciencias y Artes de Chiapas, Mexico

Doi:10.19044/esj.2019.v15n16p280 URL:http://dx.doi.org/10.19044/esj.2019.v15n16p280

\section{Resumen}

Este estudio analiza las propiedades psicométricas de la versión española del Trait Meta-Mood Scale (TMMS 24) con una muestra de 3,270 estudiantes, $69.9 \%$ mujeres y $30.1 \%$ hombres, de Chiapas, México. La consistencia interna para la escala global, obtenida a partir del coeficiente Alfa de Cronbach, es satisfactoria $(\alpha=.910)$. El análisis factorial exploratorio con extracción por factorización de ejes principales y rotación Varimax, mostró una estructura inicial de cuatro factores con autovalores superiores a uno que explicaron el $58.23 \%$ de la varianza. Sin embargo, al evaluar las cargas factoriales y la congruencia conceptual de los ítems, se determinó que un arreglo de tres factores (Atención a los sentimientos, Claridad emocional y Reparación de las emociones) es adecuado. Se concluye que el TMMS 24 es un instrumento fiable y válido para evaluar la Inteligencia emocional en adolescentes mexicanos.

Palabras clave: Propiedades psicométricas, inteligencia emocional, TMMS, adolescentes. 


\title{
Psychometric Properties of Trait Meta-Mood Scale TMMS-24 in Adolescents from Chiapas, Mexico
}

\author{
Jesús Ocaña Zúñiga \\ Germán Alejandro García Lara \\ Oscar Cruz Pérez. \\ Universidad de Ciencias y Artes de Chiapas, Mexico
}

\begin{abstract}
This study analyzes the psychometric properties of the Spanish version of the Trait Meta-Mood Scale (TMMS 24) with a sample of 3,270 students, $69.9 \%$ women and $30.1 \%$ men, from Chiapas, Mexico. The internal consistency for the entire scale is satisfactory $(\alpha=.910)$. The exploratory factorial analysis using main axis factorization and varimax rotation, improved an initial structure of four factors with eigenvalues greater than one that explained $58.23 \%$ of the variance. However, the factorial loads and the conceptual congruency of the items suggest that a three-factor solution (Attention to feelings, emotional clarity and repair of emotions) is adequate. It is concluded that the TMMS 24 is a reliable and valid instrument to assess emotional intelligence in Mexican adolescents.
\end{abstract}

Keywords: Psychometric properties, emotional intelligence, TMMS-24, adolescents.

\section{Introducción}

Las emociones tienen un papel sustancial en la vida de los seres humanos puesto que dan cuenta de su esfera afectiva, funcionamiento mental y adaptación al medio en que interactúan. Para Daco (1980, p. 102), "la experiencia de una emoción involucra un conjunto de cogniciones, actitudes y creencias sobre el mundo, que utiliza para valorar una situación concreta e influyen en el modo en el que se percibe dicha situación", por lo que su desarrollo es un proceso de evolución complejo, relacionado con la comunicación, la organización del apego, el desarrollo moral y el conocimiento social.

En el estudio de las emociones, la inteligencia emocional es un concepto de extrema utilidad para la comprensión de "los procesos emocionales básicos que subyacen al desarrollo de un adecuado equilibrio psicológico (...), el rol mediador de ciertas variables emocionales de las 
personas y su influencia sobre el ajuste psicológico y el bienestar personal" (Rey \& Extremera, 2012, pp. 87-88).

La inteligencia emocional, es considerada como una "capacidad de percibir con precisión, valorar y expresar emociones; la capacidad de acceder y/o generar sentimientos que faciliten el pensamiento; la capacidad de comprender las emociones y el conocimiento emocional; y la habilidad para regular las emociones para promover el crecimiento emocional e intelectual" (Mayer \& Salovey, 1997, p. 10).

En este contexto, la emergencia del concepto Inteligencia emocional ocurre a partir de los trabajos publicados por Mayer, DiPaolo \& Salovey (1990); Salovey \& Mayer (1990) y Mayer \& Salovey (1993). Los modelos con los que se explica este concepto se encuentran entre aquellos señalados como mixtos, como el de Goleman (1995); Boyatzis, Goleman \& Rhee (2000) y Baron (2000) (citados en Fernández \& Extremera, 2005) y el denominado Modelo de habilidad desarrollado por Mayer \& Salovey (1997). En los primeros, la Inteligencia emocional incluye un compendio de rasgos estables de personalidad, competencias socio-emocionales, aspectos motivacionales y diversas habilidades cognitivas. Por su parte, Mayer \& Salovey (1997) conciben la inteligencia emocional a partir del uso adaptativo de las emociones y su aplicación al pensamiento. En este esfuerzo por clarificar las aproximaciones al concepto, el propio Bar-on (2006), hace referencia a la Enciclopedia de Psicología Aplicada (Spielberger, 2004; citado en Bar-on, 2006), para informar sobre tres grandes modelos: el de Mayer \& Salovey, el cual analiza la inteligencia emocional como "la habilidad para percibir, entender, manejar y usar las emociones para facilitar el pensamiento" (p. 2); el modelo de Goleman que lo explica como una "amplia gama de competencias y habilidades que conducen a un funcionamiento ejecutivo" ( $p$. 2), y el modelo de Bar-on que "describe competencias emocionales y sociales interrelacionadas entre sí, habilidades y facilitadores que impactan la conducta inteligente" (p. 2). Como se aprecia, dichos modelos se diferencian a partir del énfasis que se otorga al ámbito socio emocional, de funcionamiento o para el desarrollo del pensamiento, aspecto este último sobre el cual se fundan algunos de los instrumentos más utilizados en su medición.

El análisis de la inteligencia emocional, muestra su relación con distintas variables como la Empatía (Perdomo, Pérez \& Ibáñez, 2011), la Satisfacción de vida (Fernández, Extremera \& Ramos, 2004; Fitness \& Curtis, 2005; Thompson et al., 2007; Extremera, Durán \& Rey, 2009; Delhom et al., 2017); el Ajuste psicológico, Confianza y Competencia percibida (Jiménez \& López-Zafra, 2008; Salguero, Fernández-Berrocal, Ruiz, Castillo \& Palomera, 2011); el Autoconcepto (Matalinares et al, 2005; Jiménez \& López-Zafra, 2008); las Habilidades sociales (Zabala, Valadez \& Vargas, 2008); los Estados afectivos (Merchán et al., 2018,) y en relación inversa con la Depresión 
(Aradilla, Tomás \& Gómez, 2013; Bugay, Aksöz \& Erdur, 2014); y la Ansiedad (Díaz-Castela et al, 2013). De manera particular, Jiménez-Morales \& López-Zafra (2009), Nasir \& Masrur (2010) y Fernández, Salamonson \& Griffiths (2012) hacen énfasis en que la Inteligencia emocional puede ser determinante en el éxito académico de los jóvenes estudiantes, así como en su adaptación escolar. Por su parte, Ferragout \& Fierro (2012) coinciden en que la inteligencia emocional influye en el Rendimiento académico y correlaciona con los niveles de Bienestar personal.

Existen diferentes instrumentos que evalúan la Inteligencia emocional los cuales pueden referirse como medidas de autoinforme o pruebas de habilidad. Los primeros exploran dicho constructo a través de afirmaciones que posibilitan una valoración subjetiva de las competencias emocionales; mientras que las pruebas de habilidad, examinan dichas habilidades mediante ejercicios (González, Peñalver \& Bresó, 2011).

Entre las medidas de autoinforme para evaluar la Inteligencia emocional se encuentran el Emotional Quotient Inventory (EQ-i) (Bar-On, 2006); la Wong and Law's Emotional Intelligence Scale (WLEIS) (Wong \& Law, 2002); el Trait Emotional Intelligence Questionnaire (TEIQue, Petrides \& Furnham, 2003) y el Trait Meta-Mood Scale (Salovey, Mayer, Goldman, Turvey \& Palfai, 1995). Éste último se trata de una escala de 48 ítems cuyo análisis factorial, realizado con una muestra de 200 participantes en Estados Unidos, evidenció tres factores: Percepción $(\alpha=0.86)$, Comprensión $(\alpha=0.87)$ y Regulación emocional $(\alpha=0.82)$. La propuesta de Salovey et al. (1995) recupera información de la propia percepción que el sujeto tiene de sus habilidades para atender, discriminar y reparar los propios estados emocionales que Salovey, Stroud, Woolery \& Epel (2002), en un estudio realizado con 104 universitarios estadounidenses, denominan como Índice de Inteligencia emocional percibida. Una primera adaptación al español de esta escala fue realizada por Fernández et al., en 1998; y en el 2004 fue validada en población española por Fernández, et al. (2004), con una muestra por conveniencia de 292 jóvenes universitarios obteniendo, mediante análisis factorial con extracción de componentes principales y rotación Varimax, una versión de 24 ítems del Trait Meta-Mood Scale: TMMS 24. La reducción de ítems se realizó atendiendo el criterio de cargas factoriales $\geq .400$ en la solución rotada, siendo la varianza explicada de 58.8\% y el Alpha de Cronbach superior a .85 para las tres subescalas conformadas. Esta versión de 24 ítems es la que se utilizó en este trabajo debido a su fácil aplicación, las dimensiones que evalúa y los antecedentes disponibles de aplicaciones, adaptaciones y validaciones en poblaciones de habla hispana (Cerón, Pérez-Olmos \& Ibáñez, 2011; Espinoza-Venegas et al., 2015; Taramuael y Zapata, 2017), que permiten su comparación en razón al contexto, no así por tamaños muestrales. 
Diversos estudios han explorado las propiedades psicométricas de la TMMS 24, recientemente Espinoza, Sanhueza, Ramírez \& Sáez (2015) con una muestra de 349 estudiantes de Chile, con edades de 17 a 39 años, obtuvieron una consistencia interna para la dimensión Atención $\alpha=.88$, para Compresión $\alpha=0.89$ y para Regulación $\alpha=0.86$, con valores Eigen que explican el 56.5\% de la varianza. En otro trabajo realizado con 1,417 estudiantes de enfermería y enfermeras de España con edad promedio de $\mathrm{M}=23.4$ años, Aradilla et al. (2013), confirmaron tres factores como en la propuesta de la escala original, reportando un $\alpha=0.86$ para el factor Atención, $\alpha=0.86$ para el factor Claridad y $\alpha=0.84$ para el factor Reparación. En el trabajo de Bugay et al. (2014), realizado con 387 estudiantes universitarios de Turquía con edad promedio de $\mathrm{M}=21.77$ años, reportaron para Atención un $\alpha=0.76$, para Compresión un $\alpha=0.73$ y para Regulación un $\alpha=0.60$. En el estudio de DíazCastela et al. (2013), llevado a cabo con 425 adolescentes españoles de 12 a 19 años de edad, se obtuvo para Atención y Claridad emocional un $\alpha=0.86$ respectivamente y para Regulación un $\alpha=0.81$. Por su parte, en el trabajo de Limonero, Gómez, Fernández \& Tomás (2013), realizado con 146 estudiantes universitarios de 18 a 26 años, la consistencia interna fue para Atención $\alpha=.91$, Claridad $\alpha=.89$ y Reparación $\alpha=.83$. Dichos resultados, confirman el satisfactorio nivel de confiabilidad y validez de estructura factorial para la TMMS 24.

El análisis específico de las subescalas del TMMS 24 muestra una relación más estrecha con ciertas variables. En este sentido, la Depresión correlaciona con la dimensión Atención a los sentimientos, no así con los de Claridad y Regulación emocional (Fernández et al. 2004; Extremera \& Fernández-Berrocal, 2006; Fernández-Berrocal, Alcaide, Extremera \& Pizarro, 2006; Salguero, Palomera \& Fernández-Berrocal, 2012); la Satisfacción de vida, Relaciones interpersonales y familiares se relacionan con la Claridad y Regulación; no así con la Atención a los sentimientos (Salovey et al., 2002; Thompson, Waltz, Croyle \& Pepper, 2007; Extremera et al., 2009; Bugay et al., 2014); así como la Empatía con la Atención a los sentimientos (Fitness \& Curtis, 2005); mientras que puntajes altos en Atención emocional se asocian en sentido negativo con el Ajuste psicológico (Salguero et al. 2012) y positivamente con la Ansiedad (Salguero et al., 2012); en el caso del Bienestar subjetivo, éste se correlaciona positivamente con las subescalas Atención a los sentimientos y Claridad emocional (Prado, Lidón \& Górriz, 2018).

En México no se tienen trabajos que validen el TMMS 24 en población adolescente; de ahí que el objetivo de este estudio sea el de explorar sus propiedades psicométricas de validez y confiabilidad, con el propósito de dar certidumbre a su aplicabilidad en el contexto dado para la valoración de la Inteligencia Emocional en trabajos que involucren dicho constructo. En este 
sentido, se espera que los valores hallados sean semejantes a los reportados en otras aplicaciones del mismo instrumento en otras poblaciones de habla hispana.

\section{Método}

\section{Diseño}

El trabajo siguió un diseño no experimental, de tipo transversal ex post facto.

\section{Participantes}

Los sujetos de estudio fueron 3,270 estudiantes egresados de bachillerato de régimen público de turno matutino y vespertino. Los participantes fueron seleccionados mediante muestreo no probabilístico, por conveniencia. La aplicación se realizó a 3,345 jóvenes, pero se descartaron aquellos que se negaron a participar en la investigación y quienes no completaron en su totalidad el instrumento. La edad de los participantes oscila entre 17 y 23 años, aunque la mayoría (87\%) tiene entre 17 y 19 años, siendo 18 años la edad más frecuente. La distribución por sexo es de $69.9 \%$ mujeres y $30.1 \%$ hombres.

\section{Instrumento}

Se aplicó una versión adaptada semánticamente al contexto de este estudio de la Trait Meta-Mood Scale (TMMS) de 24 ítems, la cual está basada en la versión original de Salovey \& Meyer (1995). La escala evalúa el metaconocimiento de los estados emocionales; es decir, la conciencia de las propias emociones y la capacidad para regularlas. Los ítems se agrupan en tres factores: Atención a los sentimientos (ítems 1 al 8) que explora el grado en el que las personas creen prestar atención a sus emociones y sentimientos, Claridad emocional (ítems 9 al 16) que refiere cómo las personas creen percibir sus emociones y Reparación de las emociones (ítems 17 al 24) que indaga la creencia del sujeto en su capacidad para interrumpir y regular estados emocionales negativos y prolongar los positivos. Cada ítem incluye cinco opciones de respuesta: nunca, raramente, algunas veces, con bastante frecuencia y muy frecuentemente, con valores ordinales de 1 al 5. En la adaptación al español, Fernández et al. (2004), reportaron una consistencia interna superior al .85 para todas las subescalas. Estos valores son semejantes a los reportados por Salovey et al. (1995), autores de la versión original en inglés del instrumento aplicada a 200 jóvenes estadounidenses, quienes reportaron valores cercanos a .82 para las tres subescalas. 


\section{Procedimiento}

El estudio forma parte de un proyecto más amplio en el que se analizan diversas escalas psicosociales en estudiantes preuniversitarios, el cual fue avalado por la Dirección de Investigación y Posgrado de la Universidad de Ciencias y Artes de Chiapas mediante el Acta de registro de proyecto con número de referencia DIP-0003.

Para el estudio se realizó el análisis semántico de los ítems del TMMS 24. Dicha revisión fue llevada a cabo por dos expertos en el campo, quienes valoraron la correspondencia conceptual entre los ítems y el dominio específico a medir, así como su adecuación al contexto regional. A partir de ello, se realizó la prueba piloto del instrumento con un grupo de 30 adolescentes, cuyos resultados corroboraron la comprensión de los ítems, por lo que no se llevaron a cabo modificaciones a éstos. Una vez realizado lo anterior, se procedió a su aplicación a jóvenes adolescentes egresados de bachillerato, quienes participaron en el proceso de admisión para continuar estudios universitarios en la Universidad de Ciencias y Artes de Chiapas en los años 2014 y 2015. Los participantes fueron informados del objetivo de la aplicación del instrumento; su participación fue voluntaria y se garantizó la confidencialidad y anonimato de los datos recolectados, autorizando el uso de la información con fines de investigación mediante cartas de consentimiento informado previa aplicación. En el caso de los menores de edad, por acuerdo del Comité de Posgrado de la Facultad de Ciencias Humanas y Sociales de la UNICACH, se solicitó una carta de autorización por parte de los padres o tutores al inicio del proceso de admisión para permitir la participación de los jóvenes en este proyecto de investigación. Los datos recolectados han sido protegidos atendiendo los lineamientos establecidos en la Declaración de Helsinki.

\section{Análisis}

Los datos se capturaron en un fichero del SPSS v.21 para su análisis. Para medir la confiabilidad de los instrumentos, se calculó el coeficiente Alfa de Cronbach tanto para la escala general como en la modalidad "si se elimina el elemento" para valorar si la eliminación de algunos de los ítems permitiría mejorar considerablemente la consistencia interna de la escala. Para estimar la validez de constructo se realizó el análisis factorial exploratorio (AFE) con extracción por factorización de ejes principales y rotación Varimax. Se eligió esta rotación debido a que se buscó reducir el número de variables que tienen cargas altas en cada factor y, en correspondencia con las referencias teóricas del constructo inteligencia emocional, se presume que tales factores son independientes. En una primera instancia, en la extracción de factores se consideró a todos aquellos con autovalores superiores a uno. La distribución de los ítems en los factores identificados se realizó considerando la carga 
factorial más alta, usando como criterio que fueran superiores a .300 (Ferrando \& Anguiano, 2010). La revisión de la estructura factorial resultante se revisó y comparó respecto a la propuesta estructural del modelo original. Esta revisión evidenció que dos de los factores hallados coincidían conceptualmente con la del autor original. A partir de lo anterior, se realizó un segundo análisis factorial ajustado a tres factores para verificar las cargas factoriales y la consistencia interna de dicho arreglo.

\section{Resultados}

La consistencia interna de la escala general arrojó un coeficiente Alpha de Cronbrach de $\alpha=.910$, el cual es satisfactorio. Al obtener el mismo coeficiente en la modalidad "si el ítem fuera removido", se evidenció la conveniencia de trabajar con los 24 ítems, pues en ningún caso la eliminación de alguno de los ítems propició un valor mayor del coeficiente.

La prueba de esfericidad de Barlett resultó con $\mathrm{p} \leq .000$, lo que confirmó la factibilidad del análisis factorial. El análisis factorial con extracción por factorización de ejes principales y rotación Varimax arrojó una estructura inicial de cuatro factores con autovalores (valores eigen) superiores a uno que explican el $58.23 \%$ de la varianza (tabla 1). El gráfico de sedimentación de los componentes hace evidente que la mayor proporción de varianza explicada se logra considerando entre 3 y 4 factores (figura 1 ).

Figura 1. Gráfico de sedimentación de factores

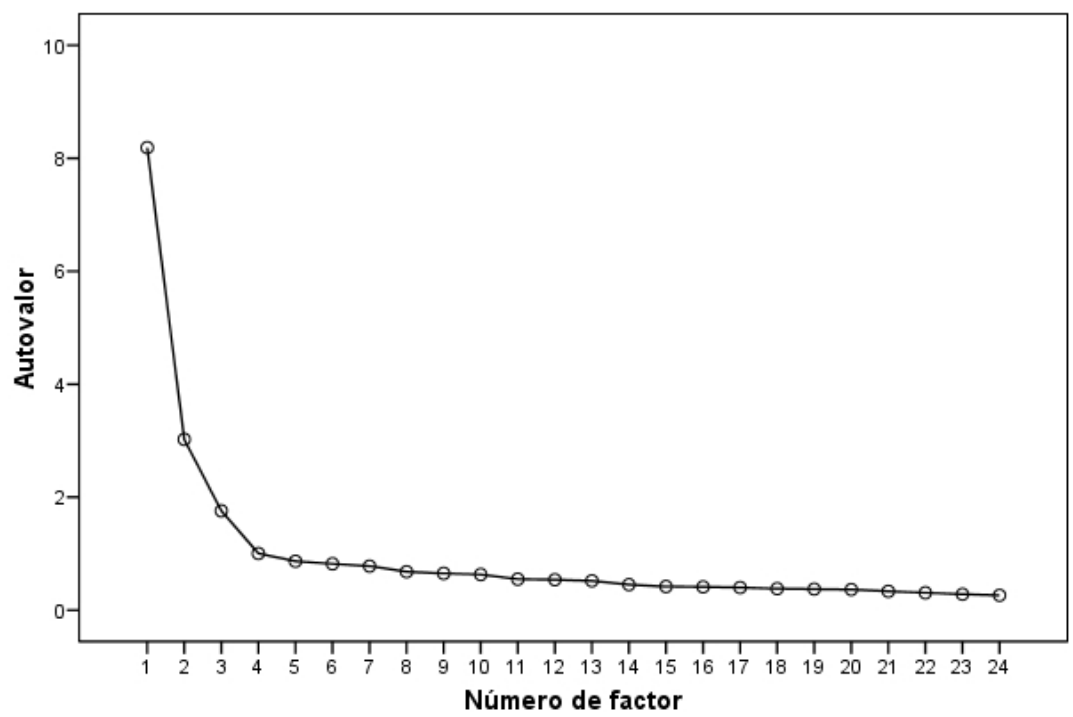

Los cuatro factores en los que se agruparon los ítems fueron los siguientes: un primer grupo de ítems se relacionan con la Atención a los sentimientos, un segundo grupo con la Claridad emocional, un tercer bloque 
nuevamente con la Atención a los sentimientos; y un cuarto grupo que se asocian con la Reparación de las emociones. La consistencia interna de las cuatro subescalas tuvo valores satisfactorios superiores a $\alpha=740$. Debido a que los ítems del factor 1 y 3 se agruparon en torno a la Atención de los sentimientos y mostraron varianzas explicativas semejantes, se rehizo el análisis factorial acotando la extracción a tres factores, utilizando las mismas especificaciones procedimentales. Esto se realizó con el propósito de evaluar un arreglo que correspondiera a la propuesta original del instrumento y, de esta manera, abrir la posibilidad de comparación de resultados con otros estudios. La solución rotada se muestra en la tabla 1.

Tabla 1. Análisis factorial extracción de factores principales, rotación Varimax, solución de tres factores.

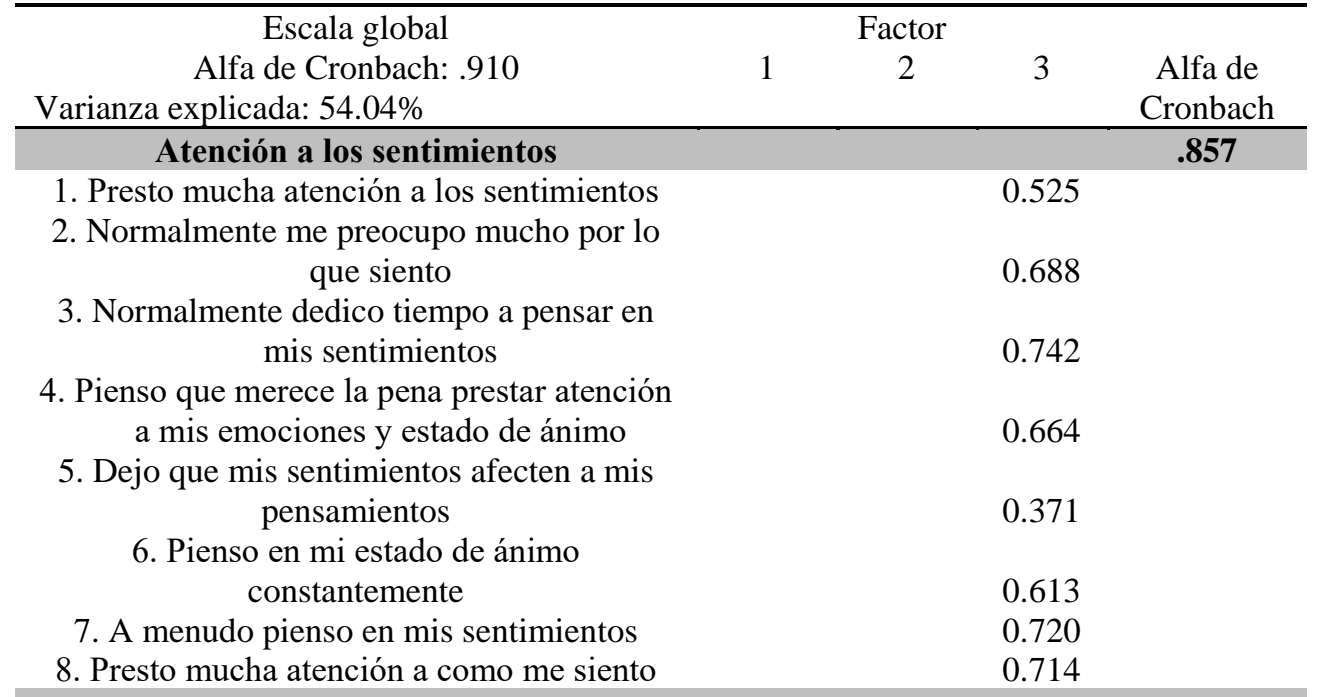

Claridad emocional

9. Tengo claro mis sentimientos

0.656

10. Frecuentemente puedo definir mis sentimientos

0.721

11.Casi siempre sé cómo me siento

0.745

12. Normalmente conozco mis sentimientos sobre las personas

0.663

13. A menudo me doy cuenta de mis sentimientos en diferentes situaciones $\quad 0.608$

14. Siempre puedo decir cómo me siento $\quad 0.685$

15. A veces puedo decir cuáles son mis emociones

16. Puedo llegar a comprender mis sentimientos

\section{Reparación de las emociones}

17. Aunque a veces me siento triste, suelo tener una visión optimista

18. Aunque me sienta mal procuro pensar en cosas agradables 
19. Cuando estoy triste, pienso en todos los placeres de la vida

20. Intento tener pensamientos positivos aunque me sienta mal

21. Si doy demasiadas vueltas a las cosas, complicándolas, trato de calmarme

22. Me preocupo por tener un buen estado de ánimo

23. Tengo mucha energía cuando me siento feliz

24. Cuando estoy enfadado intento cambiar mi estado de ánimo

0.489

Los resultados hacen evidente que la solución de tres factores es adecuada, pues los ítems coinciden con la propuesta del autor original, con valores de saturación superiores a .300 , valor considerado como el mínimo en la interpretación de las cargas factoriales (Ferrando \& Anguiano, 2010). La consistencia interna de todas las subescalas, evaluada a través de coeficiente alfa de Cronbach, es satisfactoria, en todos los casos, superiores a .840. Por tanto, se puede afirmar que este arreglo de ítems es consistente, homogéneo y que las tres subescalas miden de manera adecuada la dimensión y, en consecuencia, pueden considerarse fiables y válidas para medir el constructo de Inteligencia emocional en jóvenes preuniversitarios en México.

\section{Discusión}

Los resultados confirman la alta confiabilidad del TMMS 24 para su aplicación en el contexto de estudio. Si bien se obtienen cuatro subescalas en el análisis factorial inicial del instrumento, es evidente que, dado que los factores presentan varianzas semejantes y modestas, convergen en la misma conformación conceptual y por tanto, el arreglo de tres factores resulta pertinente. Con ello, se confirman las adecuadas propiedades psicométricas del instrumento y del modelo teórico de la inteligencia emocional propuesto por los autores de la escala (Salovey et al., 1995) y en específico de la adaptación a la versión en castellano (Fernández et al. (2004), lo que asegura la validez transcultural tanto del instrumento como del modelo teórico (Espinoza et al. (2015). Los valores de confiabilidad también son semejantes a los reportados en dicha adaptación, cercanos a .850 .

En este trabajo, el ítem 5, Dejo que mis sentimientos afecten a mis pensamientos, obtuvo un índice de saturación menor a .300 , aunque por ser cercano a éste, se consideró apropiado discriminar la diferencia. Un resultado similar se obtuvo en los trabajos de Martín-Albo, Núñez \& León (2010); Aradilla et al. (2013) y el de Pedrosa, Suárez, Lozano, Muñiz \& García (2014), por lo que habría que considerar, a criterio, su inclusión o no en la dimensión de Atención a los sentimientos al utilizar el instrumento. 
El estudio presenta información útil acerca del constructo de Inteligencia emocional. La escala analizada es de fácil aplicación y económica, por lo que puede ser utilizada para la medición de la Inteligencia emocional en población adolescente que corresponda a un contexto semejante al de los participantes de este trabajo. Tal como lo plantean Pérez, Ortega, Rincón, García \& Romero (2013), en trabajos de valoración psicométrica de escalas, es conveniente explorar nuevas metodologías y emplear muestras diferentes que permitan profundizar y ratificar los resultados reportados en este tipo de estudios. En este sentido, se plantea replicar el trabajo considerando muestras heterogéneas a fin de contar con información acerca de la capacidad de medición de este instrumento en adolescentes de contextos diversos, por ejemplo, población indígena; la cual se encuentra en una condición de alta vulnerabilidad social en Chiapas, México. En el campo disciplinar de la psicología clínica, educativa y social, la aplicación del TMMS 24 puede ser utilizada para el diseño de estrategias de intervención ante problemáticas psicosociales en adolescentes, prevención de conductas delictivas, prevención del uso de sustancias adictivas y, en general, todas aquellas áreas relacionadas con la Inteligencia Emocional y correlatos tales como la Depresión, Ansiedad, Satisfacción de vida, Bienestar Subjetivo, Relaciones Interpersonales y Empatía.

\section{References:}

1. Aradilla-Herrero, A., Tomás-Sábado, J. \& Gómez-Benito, J. (2013). Perceived emotional intelligence in nursing: psychometric properties of the Trait Meta-Mood Scale. Journal of Clinical Nursing, 23, 955966. DOI: 10.1111/jocn.12259.

2. Bar-on, R. (2000). Emotional and social intelligence: Insights from the emotional quotient inventory. En: R. Bar-On \& J. D. A. Parker (Eds.). Handbook of Emotional Intelligence (pp. 363-388). San Francisco: Jossey-Bass.

3. Bar-On, R. (2006). The Bar-On model of emotional-social intelligence (ESI). Psicothema, 18, supl., 13-25.

4. Boyatzis, R., Goleman, D. \& Rhee, K. (2000). Clustering competence in emotional intelligence: Insights from the emotional competence inventory (ECI). En: R. Bar-On \& J. D. A. Parker (Eds.). Handbook of emotional intelligence (pp. 343-362). San Francisco: Jossey-Bass.

5. Bugay, A., Aksöz, I., \& Erdur Baker, O. (2014). Examining the reliability and validity of the Turkish version of the Trait Meta-Mood Scale. International Journal of Human Sciences, 11(2), 1264-1273. DOI: 10.14687/ijhs.v11i2.3007. 
6. Cerón D., Pérez-Olmos I. \& Ibáñez M. (2011). Inteligencia emocional en adolescentes de dos colegios de Bogotá. Revista Colombiana de Psiquiatría, 40(1), 49-64.

7. Daco, P. (1980). Tu personalidad. Madrid: Daimon.

8. Delhom I., Gutiérrez, M., Lucas-Molina, B., Meléndez, J. C. (2017). Emotional intelligence in older adults: psychometric properties of the TMMS-24 and relationship with psychological well-being and life satisfaction. Int Psychogeriatr. 29(8), 1327-1334.

9. Díaz-Castela, M. del M., Hale, W. W., Muela, J. A., EspinosaFernández, L., Klimstra, T. \& García-Lopez, L. J. (2013). The measurement of Perceived Emotional Intelligence for spanish adolescents with social anxiety disorder symptoms. Anales de Psicología, mayo, 29(2), 509-515. http://dx.doi.org/10.6018/analesps.29.2.144271.

10. Espinoza-Venegas, M., Sanhueza-Alvarado, O., Ramírez-Elizondo, N. \& Sáez-Carrillo, K. (2015). Validación de constructo y confiabilidad de la escala de inteligencia emocional en estudiantes de enfermería. Rev. Latino-Am. Enfermagem, ene - feb, 23(1), 139-147. DOI: 10.1590/0104-1169.3498.2535. Recuperado de: www.eerp.usp.br/rlae

11. Extremera, N., Durán, A. \& Rey, L. (2009). The moderating effect of trait meta-mood and perceived stress on life satisfaction. Personality and Individual Differences, 47, 116-121. DOI:10.1016/j.paid.2009.02.007.

12. Extremera, N. \& Fernández-Berrocal, P. (2006). Emotional Intelligence as predictor of mental, social, and physical health in university students. The Spanish Journal of Psychology, 9 (1), 45-51.

13. Ferrando, P.J. \& Anguiano-Carrasco, C. (2010). El análisis factorial como técnica de investigación en psicología. Papeles del Psicólogo, 31(1), 18-33.

14. Fitness, J. \& Curtis, M. (2005). Emotional intelligence and the Trait Meta-Mood Scale: Relationships with empathy, attributional complexity, self-control, and responses to interpersonal conflict. $E$ Journal of Applied Psychology: Social Section, 1(1), 50-62. http://ojs.lib.swin.edu.au/index.php/ejap/article/viewFile/6/15

15. Ferragut, M. \& Fierro, A. (2012). Inteligencia emocional, bienestar personal y rendimiento académico en preadolescentes. Revista Latinoamericana de Psicología, 44(3) 95-104.

16. Fernández-Berrocal, P., Alcaide, R., Domínguez, E., FernándezMcNally, C., Ramos, N. S., \& Ravira, M. (1998). Adaptación al castellano de la escala rasgo de metaconocimiento sobre estados emocionales de Salovey et al.: datos preliminares. Libro de Actas del $V$ Congreso de Evaluación Psicológica. Málaga. 
17. Fernández-Berrocal, P., Alcaide, R., Extremera, N. \& Pizarro, D. A. (2006). The role of emotional intelligence in anxiety and depression among ad-olescents. Individual Differences Research, 4, 16-27.

18. Fernández-Berrocal, P., Extremera, N. \& Ramos, N. (2004). Validity and reliability of the Spanish modified version of the Trait Meta-Mood Scale. Psychological Reports, 94, 751-755.

19. Fernández Berrocal, P. \& Extremera Pacheco, N. (2005). La inteligencia emocional y la educación de las emociones desde el modelo de Mayer y Salovey. Revista Interuniversitaria de Formación del Profesorado, 19(3), (2005), 63-93.

20. Fernández, R., Salamonson, Y. \& Griffiths R. (2012). Emotional intelligence as a predictor of academic performance in first-year accelerated graduate entry nursing students. J Clin Nurs, 21 (23-24), 3485-92.

21. Goleman, D. (1995). Inteligencia emocional. Barcelona: Kairós.

22. González Robles, A., Peñalver González, J. \& Bresó Esteve, E. (2011). La evaluación de la inteligencia emocional: ¿autoinformes o pruebas de habilidad? Fòrum De Recerca, 16, 699-712. Recuperado de: http://repositori.uji.es/xmlui/bitstream/handle/10234/77307/fr_2011_ 7_3.pdf?sequence=1

23. Jiménez, M. I. \& López-Zafra, E. (2008). El autoconcepto emocional como factor de riesgo emocional en estudiantes universitarios: Diferencias de género y edad. Boletín de Psicología, 93, 21-39.

24. Jiménez-Morales, M. I. \& López-Zafra, E. (2009). Inteligencia emocional y rendimiento escolar: estado actual de la cuestión. Revista Latinoamericana de Psicología, 41(1), 69-79.

25. Limonero, J. T., Gómez-Romero, M. J., Fernández-Castro, J. \& Tomás-Sábado, J. (2013). Influencia de la inteligencia emocional percibida y la impulsividad en el abuso de cánnabis en jóvenes. Ansiedad y Estrés, 19 (2-3), 223-234.

26. Martín-Albo, J., Núñez, J. L. \& León, J. (2010). Analysis of the psychometric properties of the Spanish version of the Trait MetaMood Scale in a sports context. Psychological Reports 106 (2), 1-13.

27. Matalinares, M., Arenas, C., Dioses, A., Muratta, R., Pareja, C., Díaz, G., García, C., Diego, M. \& Chávez, J. (2005). Inteligencia emocional y autoconcepto en colegiales de Lima Metropolitana. Revista de Investigación en Psicología, 8(2), 41-55.

28. Mayer, J. D., DiPaolo, M. \& Salovey, P. (1990). Perceiving affective content in ambiguous visual stimuli: A component of emotional intelligence. Journal of Personality Assessment, 54, 772-781.

29. Mayer, J. D. \& Salovey, P. (1993). The intelligence of emotional intelligence. Intelligence, 17, 433-442. 
30. Mayer, J. D. \& Salovey, P. (1997). What is emotional intelligence? En: P. Salovey y D. Sluyter (Eds). Emotional development and emotional intelligence: implications for educators (pp. 3-31). New York: Basic Books.

31. Merchán, A., Morales, L., Martínez, C. \& Gil, P. (2018). Valor predictivo de la inteligencia emocional rasgo en los estados afectivos: una comparación de género. Revista INFAD de Psicología, 1(1), 137146.

32. Nasir, M. \& Masrur, R. (2010). An exploration of emotional intelligence of the students of IIUI in relation to gender, age and academic achievement. Bulletin of Education and Research, 32(1), 3751.

33. Pedrosa, I., Suárez-Álvarez, J., Lozano, L. M., Muñiz, J. \& GarcíaCueto, E. (2014). Assessing perceived emotional intelligence inadolescents: New validity evidence of Trait Meta-Mood Scale-24. Journal of Psychoeducational Assessment, 32(8), 737-746. http://dx.doi.org/10.1177/0734282914539238

34. Perdomo, C., Pérez, D. M. \& Ibáñez, I. (2011). Inteligencia emocional en adolescentes de dos colegios de Bogotá y variables asociadas. Rev Colomb. Psiquiatr, 40 (1), 49-64.

35. Pérez, O. M., Ortega, N. A., Rincón, A. B., García, R. \& Romero M. (2013). Propiedades psicométricas del cuestionario de agresión en dos muestras diferentes de Hidalgo, México. European Scientific Journal, 9(32), 107-120.

36. Petrides, K. V. \& Furnham, A. (2003). Trait emotional intelligence: behavioral validation in two studies of emotion recognition and reactivity to mood induction. European Joumal of Personality, 17,3957.

37. Prado, V., Lidón, B. \& Gorríz, A. (2018). Trait emotional intelligence and subjective well-being in adolescents: The moderating role of feelings. Psicothema, 30(3), 310-315.

38. Rey, L. \& Extremera, N. (2012). Inteligencia emocional percibida, felicidad y estrategias distractoras en adolescentes. Boletín de Psicología, marzo, 104, 87-101.

39. Salguero, J. M., Fernández-Berrocal, P., Ruiz-Aranda, D., Castillo, R. \& Palomera, R. (2011). Inteligencia emocional y ajuste psicosocial en la adolescencia: El papel de la percepción emocional. European Journal of Education and Psychology, 4(2), 143-152.

40. Salguero, J. M., Palomera, R. \& Fernández-Berrocal, P. (2012) Perceived emotional intelligence as predictor of psychological adjustment in ado-lescents: a 1-year prospective study. European Journal of Psychology of Education, 27, 1, 21-34. 
41. Salovey, P. \& Mayer, J. D. (1990). Emotional intelligence. Imagination, Cognition, and Personality, 9, 185-211.

42. Salovey, P., Mayer, J. D., Goldman, S. L., Turvey, C. \& Palfai, T. P. (1995). Emotional attention, clarity, and repair: exploring emotional intelligence using the Trait Meta-Mood Scale. En J. W. Pennebaker (Ed.). Emotion, disclosure and health (pp. 125-154). Washington: American Psychological Association.

43. Salovey, P., Stroud, L. R., Woolery, A. \& Epel, E. S. (2002). Perceived emotional intelligence, stress reactivity, and symptom reports: further explorations using the trait meta-mood scale. Psychology and Health, 17, 611-627.

44. Taramuel, J.A y Zapata, V.H. (2017). Aplicación del test TMMS-24 para el análisis y descripción de la Inteligencia Emocional considerando la influencia del sexo. Revista Publicando, 4(11/1), 162181.

45. Thompson, B. L., Waltz, J., Croyle, K. \& Pepper, A. C. (2007). Trait meta-mood and affect as predictors of somatic symptoms and life satisfaction. Personality and Individual Differences, 43, 1786-1795. DOI:10.1016/j.paid.2007.05.017

46. Wong, C. S. \& Law, K. S. (2002). The effects of leader and follower emotional intelligence on performance and attitude: an exploratory study. The Leadership Quarterly, $\mathrm{n}^{\mathrm{0}} 13,243-274$.

47. Zabala, M. A., Valadez, M. D. \& Vargas, M. C. (2008). Inteligencia emocional y habilidades sociales en adolescentes con alta aceptación social. Electronic Journal of Research in Educational Psychology, 15(6), 319-338. 